УДК 517.98

\title{
The Uniqueness of the Translation-invariant Gibbs Measure for Four State HC-models on a Cayley Tree
}

\author{
Rustam M. Khakimov* \\ Institute of Mathematics \\ Do'rmon Yo'li str., 29, Tashkent, 100125 \\ Uzbekistan
}

Received 04.01.2015, received in revised form 17.02.2015, accepted 06.03.2015

We consider fertile Hard-Core (HC) models with activity parameter $\lambda>0$ and four states on the Cayley tree of order two. It is known that there are three types of such models. In this paper for each of these models the uniqueness of the translation-invariant Gibbs measure is proved.

Keywords: Cayley tree, configuration, HC-model, Gibbs measure, translation-invariant measures.

\section{Introduction}

The study of limiting Gibbs measures play an important role in many fields of science. The hard core (HC) model arises in the study of random independent sets of a graph ( [1]), the study of gas molecules on a lattice [2], and in the analysis of multi-casting in telecommunication networks [3].

A HC model on $d$-dimensional lattice $\mathbb{Z}^{d}$, was introduced and studied by Mazel and Suhov in $[4]$.

The description of all limiting Gibbs measures for a given Hamiltonian is one of main problems in the theory of Gibbs measures. (see f.e. [5-7]).

In [8] a HC (Hard Core) model with two states on a Cayley tree was studied and it was proved that the translation-invariant Gibbs measure for this model is unique. Moreover, it was proved non uniqueness of periodic Gibbs measures of period two for some conditions on parameters. In [9] weekly periodic Gibbs measure for the two state HC-model is investigated and it is shown that the weekly periodic measure is unique.

Works [10,11] are devoted to Gibbs measures for three state HC-models on a Cayley tree of order $k \geqslant 1$. In [12] the fertile three-state HC-models corresponding to graphs "the hinge", "the pipe", "the wand", "the key" and four-state HC-models corresponding to graphs "the stick", "the key", "the gun" are introduce. In [10] translation-invariant and periodic Gibbs measures for HC-model in the case "the key" on a Cayley tree is studied and it was proved that the translation-invariant measure is unique for any positive activity $\lambda$. In [11] translation-invariant and periodic Gibbs measures for HC-model in cases "the pipe", "the hinge", "the wand" are studied. In [13] translation-invariant Gibbs measures for three state HC-models on a Cayley tree of order three are considered and the exact critical values of the parameter $\lambda$ are found such that for activities larger than these critical values the measure is not unique.

In this paper we consider fertile four states HC-models corresponding to graphs "the stick", "the key" and "the gun" on a Cayley tree of order two. In each case it is proved that the translation-invariant Gibbs measure is unique.

*rustam-7102@rambler.ru

(C) Siberian Federal University. All rights reserved 


\section{Definitions and known facts}

The Cayley tree $\Im^{k}$ of order $k \geqslant 1$ is an infinite tree, i.e., a graph without cycles, such that exactly $k+1$ edges originate from each vertex. Let $\Im^{k}=(V, L, i)$, where $V$ is the set of vertices $\Im^{k}, L$ the set of edges and $i$ is the incidence function setting each edge $l \in L$ into correspondence with its endpoints $x, y \in V$. If $i(l)=\{x, y\}$, then the vertices $x$ and $y$ are called the nearest neighbors, denoted by $l=\langle x, y\rangle$. The distance $d(x, y), x, y \in V$ on the Cayley tree is the number of edges of the shortest path from $x$ to $y$ :

$$
d(x, y)=\min \left\{d \mid \exists x=x_{0}, x_{1}, \ldots, x_{d-1}, x_{d}=y \in V \text { such that }\left\langle x_{0}, x_{1}\right\rangle, \ldots,\left\langle x_{d-1}, x_{d}\right\rangle\right\} .
$$

For a fixed $x^{0} \in V$ we set $W_{n}=\left\{x \in V \mid d\left(x, x^{0}\right)=n\right\}, V_{n}=\left\{x \in V \mid d\left(x, x^{0}\right) \leqslant n\right\}, \quad L_{n}=$ $\left\{l=\langle x, y\rangle \in L \mid x, y \in V_{n}\right\}$.

We consider the four-state nearest-neighbor HC-model on a Cayley tree. In this model each vertex $x$ is assigned one of the values $\sigma(x) \in\{0,1,2,3\}$. The values $\sigma(x)=1,2,3$ mean that the vertex $x$ is "occupied" and the value $\sigma(x)=0$ means that $x$ is "vacant".

A configuration $\sigma=\{\sigma(x), x \in V\}$ on a Cayley tree is given by a function from $V$ to $\{0,1,2,3\}$. The set of all configurations in $V$ denote by $\Omega$. Configurations in $V_{n}\left(W_{n}\right)$ is defined analogously and the set of all configurations in $V_{n}\left(W_{n}\right)$ denote by $\Omega_{V_{n}}\left(\Omega_{W_{n}}\right)$.

Here we consider three types of fertile graphs (see Definition 2) with four vertices $0,1,2,3$ (on the set of values $\sigma(x)$ ) which have the forms:

$$
\begin{array}{ll}
\text { stick: } & \{0,1\}\{1,2\}\{2,3\} \\
\text { key }: & \{0,1\}\{0,2\}\{1,2\}\{2,3\} \\
\text { gun }: & \{0,1\}\{0,2\}\{1,2\}\{2,2\}\{2,3\} .
\end{array}
$$

Graphs that are not fertile are said to be sterile (see. [12]).

Let $O=\{$ the stick, the key, the gun $\}, G \in O$. We say that a configuration $\sigma$ is $G$-admissible configuration on a Cayley tree (in $V_{n}$ or $W_{n}$ ), if $\{\sigma(x), \sigma(y)\}$ is an edge of $G$ for any pair of nearestneighbors $x, y$ in $V$ (in $V_{n}$ ). We let $\Omega^{G}\left(\Omega_{V_{n}}^{G}\right)$ denote the set of $G$-admissible configurations.

The set of activity of graph $G$ is a function $\lambda: G \rightarrow R_{+}\left(R_{+}\right.$denotes the positive real numbers) [12]. The value $\lambda_{i}$ of function $\lambda$ at a vertex $i \in\{0,1,2,3\}$ is called the activity of $\lambda$.

Given $G$ and $\lambda$, we define the $G-\mathrm{HC}$ Hamiltonian as

$$
H_{G}^{\lambda}(\sigma)= \begin{cases}\sum_{x \in V} \log \lambda_{\sigma(x)}, & \text { if } \sigma \in \Omega^{G}, \\ +\infty, & \text { if } \sigma \notin \Omega^{G} .\end{cases}
$$

We write $x<y$, if a path from $x^{0}$ to $y$ passes through $x$. A vertex $y$ is called a direct descendant of $x$ if $y>x$ and $x, y$ are neighbors. We let $S(x)$ denote the set of direct descendants of $x$. We note that in $\Im^{k}$, any vertex $x \neq x^{0}$ has $k$ direct descendants and the vertex $x^{0}$ has $k+1$ descendants.

For $\sigma_{n} \in \Omega_{V_{n}}^{G}$ we set

$$
\# \sigma_{n}=\sum_{x \in V_{n}} \mathbf{1}\left(\sigma_{n}(x) \geqslant 1\right)
$$

which is the number of occupied vertices in $\sigma_{n}$.

Let $z: x \mapsto z_{x}=\left(z_{0, x}, z_{1, x}, z_{2, x}, z_{3, x}\right) \in R_{+}^{4}$ be a vector-valued function on $V$. For $n=$ $1,2, \ldots$ and $\lambda>0$ we consider the probability measure $\mu^{(n)}$ on $\Omega_{V_{n}}^{G}$, defined as

$$
\mu^{(n)}\left(\sigma_{n}\right)=\frac{1}{Z_{n}} \lambda^{\# \sigma_{n}} \prod_{x \in W_{n}} z_{\sigma(x), x}
$$


Here $Z_{n}$ is normalizing divider

$$
Z_{n}=\sum_{\widetilde{\sigma}_{n} \in \Omega_{V_{n}}^{G}} \lambda^{\# \widetilde{\sigma}_{n}} \prod_{x \in W_{n}} z_{\tilde{\sigma}(x), x}
$$

We say, that the probability measure $\mu^{(n)}$ is consistent if for any $n \geqslant 1$ and $\sigma_{n-1} \in \Omega_{V_{n-1}}^{G}$ :

$$
\sum_{\omega_{n} \in \Omega_{W_{n}}} \mu^{(n)}\left(\sigma_{n-1} \vee \omega_{n}\right) \mathbf{1}\left(\sigma_{n-1} \vee \omega_{n} \in \Omega_{V_{n}}^{G}\right)=\mu^{(n-1)}\left(\sigma_{n-1}\right)
$$

In this case, there exists a unique probability measure $\mu$ on $\left(\Omega^{G}, \mathbf{B}\right)$ such that for any $n$ and $\sigma_{n} \in \Omega_{V_{n}}^{G}$

$$
\mu\left(\left\{\left.\sigma\right|_{V_{n}}=\sigma_{n}\right\}\right)=\mu^{(n)}\left(\sigma_{n}\right),
$$

where $\mathbf{B}$ is $\sigma$-algebra generated by cylindrical subsets $\Omega^{G}$.

Definition 1. The measure $\mu$ defined by (1) with condition (2) is called (G-)HC-Gibbs measure with $\lambda>0$, associated to the function $z: x \in V \backslash\left\{x^{0}\right\} \mapsto z_{x}$.

The set of such measures (for all sorts of $z$ ) denoted by $\mathcal{S}_{G}$.

A Gibbs measure is called translation-invariant if it corresponding to a constant function $z_{x} \equiv z$.

Definition 2 ( [12]). If there is a set of activities $\lambda$ on $G$ such that the corresponding $G-H C$ Hamiltonian has at last two translation-invariant Gibbs measures, then the graph $G$ is called a fertile graph.

Let $L(G)$ be the set of edges of $G$. We let $A \equiv A^{G}=\left(a_{i j}\right)_{i, j=0,1,2,3}$ denote the incidence matrix of $G$, i.e.

$$
a_{i j} \equiv a_{i j}^{G}= \begin{cases}1, & \text { if }\{i, j\} \in L(G) \\ 0, & \text { if }\{i, j\} \notin L(G)\end{cases}
$$

The next theorem states a condition on $z_{x}$ that guarantees that the measure $\mu^{(n)}$ is consistent.

Theorem 1 ( [14]). Probability measures $\mu^{(n)}, n=1,2, \ldots$, defined by (1), are consistent if and only if the equalities

$$
\begin{aligned}
& z_{0, x}^{\prime}=\lambda \prod_{y \in S(x)} \frac{a_{10} z_{0, y}^{\prime}+a_{11} z_{1, y}^{\prime}+a_{12} z_{2, y}^{\prime}+a_{13}}{a_{30} z_{0, y}^{\prime}+a_{31} z_{1, y}^{\prime}+a_{32} z_{2, y}^{\prime}+a_{33}}, \\
& z_{1, x}^{\prime}=\lambda \prod_{y \in S(x)} \frac{a_{20} z_{0, y}^{\prime}+a_{21} z_{1, y}^{\prime}+a_{22} z_{2, y}^{\prime}+a_{23}}{a_{30} z_{0, y}^{\prime}+a_{31} z_{1, y}^{\prime}+a_{32} z_{2, y}^{\prime}+a_{33}}, \\
& z_{2, x}^{\prime}=\lambda \prod_{y \in S(x)} \frac{a_{30} z_{0, y}^{\prime}+a_{31} z_{1, y}^{\prime}+a_{32} z_{2, y}^{\prime}+a_{33}}{a_{30} z_{0, y}^{\prime}+a_{31} z_{1, y}^{\prime}+a_{32} z_{2, y}^{\prime}+a_{33}},
\end{aligned}
$$

(where $\left.z_{i, x}^{\prime}=\lambda z_{i, x} / z_{3, x}, \quad i=0,1,2\right)$ hold for any $x \in V$.

\section{Translation-invariant Gibbs measures}

We assume that $z_{3, x} \equiv 1$ and $z_{i, x}=z_{i, x}^{\prime}>0, \quad i=0,1,2$. Then for any functions $x \in V \mapsto$ $z_{x}=\left(z_{0, x}, z_{1, x}, z_{2, x}\right)$, satisfying the relation 


$$
z_{i, x}=\lambda \prod_{y \in S(x)} \frac{a_{i 0} z_{0, y}+a_{i 1} z_{1, y}+a_{i 2} z_{2, y}+a_{i 3}}{a_{30} z_{0, y}+a_{31} z_{1, y}+a_{32} z_{2, y}+a_{33}}, \quad i=0,1,2,
$$

there exist a unique $G$-HC Gibbs measure $\mu$ and vice versa. We consider the translation-invariant solutions such that $z_{x}=z \in R_{+}^{3}, x \neq x_{0}$. In cases $G=$ stick, $G=$ key and $G=$ gun from (3) we get following systems of equations

$$
\begin{gathered}
\left\{\begin{array}{c}
z_{0}=\lambda\left(\frac{z_{1}}{z_{2}}\right)^{k}, \\
z_{1}=\lambda\left(\frac{z_{0}+z_{2}}{z_{2}}\right)^{k}, \\
z_{2}=\lambda\left(\frac{z_{1}+1}{z_{2}}\right)^{k},
\end{array}\right. \\
\left\{\begin{array}{c}
z_{0}=\lambda\left(\frac{z_{1}+z_{2}}{z_{2}}\right)^{k}, \\
z_{1}=\lambda\left(\frac{z_{0}+z_{2}}{z_{2}}\right)^{k}, \\
z_{2}=\lambda\left(\frac{z_{0}+z_{1}+1}{z_{2}}\right)^{k} \\
z_{0}=\lambda\left(\frac{z_{1}+z_{2}}{z_{2}}\right)^{k}, \\
z_{1}=\lambda\left(\frac{z_{0}+z_{2}}{z_{2}}\right)^{k}, \\
z_{2}=\lambda\left(\frac{z_{0}+z_{1}+z_{2}+1}{z_{2}}\right)^{k}
\end{array}\right.
\end{gathered}
$$

respectively.

Lemma. If $\left(z_{0}, z_{1}, z_{2}\right)$ is a solution of the system of equations (5) or (6) then $z_{0}=z_{1}$.

The proof is obtained by subtracting directly from the first equation of the second in (5) and (6), respectively.

The following statement gives estimates for any solution of the system of equations (4).

Statement 1. If $z=\left(z_{0}, z_{1}, z_{2}\right)$ is the solution of (4), then

$$
\begin{aligned}
& \text { 1) } \frac{\lambda^{k+1}}{\sqrt[k+1]{\lambda^{k}\left(2^{k} \lambda+1\right)^{k^{2}}}}<z_{0}<\frac{2^{k^{2}} \lambda^{k+1}}{\sqrt[k+1]{\lambda^{k}(\lambda+1)^{k^{2}}}} \\
& \text { 2) } \lambda<z_{1}<2^{k} \lambda \\
& \text { 3) } \sqrt[k+1]{\lambda(\lambda+1)^{k}}<z_{2}<\sqrt[k+1]{\lambda\left(2^{k} \lambda+1\right)^{k}}
\end{aligned}
$$

Proof. 1. The first inequality follows from the first equation (4) using estimates for $z_{1}$ and $z_{2}$.

2. From the second equation of (4) we obtain that $z_{1}>\lambda$. Dividing the first equation to the third we will have

$$
\frac{z_{0}}{z_{2}}=\left(\frac{z_{1}}{z_{1}+1}\right)^{k}<1
$$

Using the last inequality from the second equation follows $z_{1}<2^{k} \lambda$.

3. Using estimate for $z_{1}$, inequality obtained from the third equation of (4) we obtain $z_{2}=$ $\sqrt[k+1]{\lambda\left(z_{1}+1\right)^{k}}$

\section{The case $\mathrm{G}=$ stick}

In the case stick we have the following 
Theorem 2. In the case $G=$ stick for $k=2$ and $\lambda>0$ there is a unique HC translationinvariant Gibbs measure.

Proof. We find $z_{2}$ from the third equation of system (4) for $k=2$ and substitute into the first equation. Using obtained expressions for $z_{2}$ and $z_{0}$ from the second equation we get

$$
z_{1}=\lambda \cdot\left(\frac{z_{1}^{2}}{\left(z_{1}+1\right)^{2}}+1\right)^{2}=f\left(z_{1}\right)
$$

Rewrite the equation (7) in the form

$$
\lambda=\frac{z_{1}}{\left(\frac{z_{1}^{2}}{\left(z_{1}+1\right)^{2}}+1\right)^{2}}=\psi\left(z_{1}\right) .
$$

It is easy to see that the function $\psi\left(z_{1}\right)$ is strictly increasing for $z_{1}>0$, consequently for any value $\lambda>0$ there exists a unique value $z_{1}$, i.e. the equation $z_{1}=f\left(z_{1}\right)$ has a unique solution for $\lambda>0$.

\section{The case $G=$ key}

In the case denoting $\sqrt[k]{z_{1}}=x>0, \sqrt[k]{z_{2}}=y>0, \sqrt[k]{\lambda}=a$ and using lemma, from the system of equations (5) we obtain the following system of equations

$$
\left\{\begin{array}{l}
x=a \cdot \frac{x^{k}+y^{k}}{y^{k}}=a \cdot\left(\frac{x}{y}\right)^{k}+a, \\
y=a \cdot \frac{2 x^{k}+1}{y^{k}}=2 a \cdot\left(\frac{x}{y}\right)^{k}+\frac{a}{y^{k}} .
\end{array}\right.
$$

From the first equation of (9) we find $\left(\frac{x}{y}\right)^{k}=\frac{x-a}{a}$ and substitute into second equation we get $y=2 x-2 a+\frac{a}{y^{k}}$ or

$$
x=\frac{y}{2}-\frac{a}{2 y^{k}}+a .
$$

Using the last expression for $x$, from the second equation of (9) we obtain

$$
y^{k+1}=a \cdot\left[2\left(\frac{y}{2}-\frac{a}{2 y^{k}}+a\right)^{k}+1\right] .
$$

This equation is equivalent to the equation

$$
f(y)=2 y^{7}-a y^{6}-4 a^{2} y^{5}-\left(4 a^{3}+2 a\right) y^{4}+2 a^{2} y^{3}+4 a^{3} y^{2}-a^{3}=0,
$$

for $k=2$ which by the known theorem of Descartes up to three positive solutions. Moreover, $f(0)=-a^{3}<0$ and $f(y) \rightarrow+\infty$ for $y \rightarrow+\infty$, i.e. the equation $f(y)=0$ has at least one positive solution.

Thus, we have the following

Statement 2. The system of equations (9) has at least one and at most three solutions for $k=2$.

We show that the system (9) has only one solution for any values $a>0$. It is easy to see that the equation $z_{1}=f\left(z_{1}\right)$ has more than one solution if and only if the equation $z_{1} f^{\prime}\left(z_{1}\right)=f\left(z_{1}\right)$ 
has more than one solution. We use this property twice. From the system of equations (5) for $k=2$ we get the equation

$$
z_{1}=\lambda \cdot\left(\frac{z_{1}+\sqrt[3]{\lambda\left(2 z_{1}+1\right)^{2}}}{\sqrt[3]{\lambda\left(2 z_{1}+1\right)^{2}}}\right)^{2}=\lambda \cdot\left(\frac{z_{1}}{\sqrt[3]{\lambda\left(2 z_{1}+1\right)^{2}}}+1\right)^{2}=f\left(z_{1}\right)
$$

We calculate the derivative

$$
f^{\prime}\left(z_{1}\right)=2 \lambda\left(\frac{z_{1}}{\sqrt[3]{\lambda\left(2 z_{1}+1\right)^{2}}}+1\right) \frac{2 z_{1}+3}{\sqrt[3]{\lambda\left(2 z_{1}+1\right)^{5}}} .
$$

Then from $z_{1} f^{\prime}\left(z_{1}\right)=f\left(z_{1}\right)$ we obtain

$$
z_{1}=\frac{\sqrt[3]{\lambda\left(2 z_{1}+1\right)^{5}}}{2 z_{1}+5}=\varphi\left(z_{1}\right)
$$

Taking the derivative

$$
\varphi^{\prime}\left(z_{1}\right)=\frac{4 \lambda\left(2 z_{1}+1\right)\left(2 z_{1}+11\right)}{3\left(2 z_{1}+5\right)^{2} \sqrt[3]{\lambda^{2}\left(2 z_{1}+1\right)}},
$$

we consider the equation $z_{1} \varphi^{\prime}\left(z_{1}\right)=\varphi\left(z_{1}\right)$. It is equivalent to the following quadratic equation

$$
4 z_{1}^{2}-8 z_{1}+15=0
$$

that has no real roots. Hence, the equation $z_{1}=f\left(z_{1}\right)$ has unique solution. Consequently by Statement 2, we have

Theorem 3. In the case $G=k e y$ for $k=2$ and $\lambda>0$ Hard-Core translation-invariant Gibbs measure is a unique.

\section{The case $\mathrm{G}=$ gun}

By the lemma from (6) we obtain the following system of equations

$$
\left\{\begin{array}{l}
x=a \cdot \frac{x^{k}+y^{k}}{y^{k}}=a \cdot\left(\frac{x}{y}\right)^{k}+a, \\
y=a \cdot \frac{2 x^{k}+y^{k}+1}{y^{k}}=2 a \cdot\left(\frac{x}{y}\right)^{k}+\frac{a}{y^{k}}+a,
\end{array}\right.
$$

where $\sqrt[k]{z_{1}}=x>0, \sqrt[k]{z_{2}}=y>0, \sqrt[k]{\lambda}=a$.

It is clear that $x>a$. Similarly to the previous case from (10) we have

$$
y^{k+1}=2 a \cdot\left(\frac{y}{2}-\frac{a}{2 y^{k}}+\frac{a}{2}\right)^{k}+a y^{k}+a .
$$

For $k=2$ we transform this equation to

$$
f(y, a)=2 y^{7}-3 a y^{6}-2 a^{2} y^{5}-\left(a^{3}+2\right) y^{4}+2 a^{2} y^{3}+2 a^{3} y^{2}-a^{3}=0 .
$$

Analyzing the last equation similar to the previous case we get the following

Statement 3. The system of equations (10) has at least one and at most three solutions for $k=2$. 
We show that the system (10) has only one solution for any values $a>0$. For this from (10) we obtain the equation

$$
x=a \cdot\left[\left(\frac{x^{3}}{2 x^{3}-a x^{2}+x-a}\right)^{2}+1\right]=f(x) .
$$

The derivative of function $f(x)$ is

$$
f^{\prime}(x)=\frac{2 a x^{5}}{\left(2 x^{3}-a x^{2}+x-a\right)^{3}}\left(-a x^{2}+2 x-3 a\right) .
$$

Transforming equation $x f^{\prime}(x)=f(x)$, we get

$$
2 x^{6}\left(-a x^{2}+2 x-3 a\right)=\left(x^{6}+\left(2 x^{3}-a x^{2}+x-a\right)^{2}\right)\left(2 x^{3}-a x^{2}+x-a\right),
$$

which has no solutions for $a>\frac{1}{\sqrt{3}}$. Hence the equation (11) by Statement 3 has only one solution for $a>\frac{1}{\sqrt{3}}$.

We consider the case $0<a \leqslant \frac{1}{\sqrt{3}}$. From (12) it follows that the function $f(x)$ has critical points $x_{1}=\frac{1-\sqrt{1-3 a^{2}}}{a}$ and $x_{2}=\frac{1+\sqrt{1-3 a^{2}}}{a}\left(a<x_{1}<x_{2}\right.$ for $\left.0<a \leqslant \frac{1}{\sqrt{3}}\right)$, decreases for $a<x<x_{1}, x>x_{2}$ and increases for $x_{1}<x<x_{2}$. Hence $x_{1}=x_{\min }$ and $x_{2}=x_{\max }$. We note, that the value of the function $f(x)$ in the point $a$ lies above the bisector $y=x$, because $f(a)=2 a>a$. Moreover, values of the function $f(x)$ in points $x_{\min }$ and $x_{\max }$ lies below the bisector $y=x$, because $f\left(x_{\min }\right)<x_{\min }$ and $f\left(x_{\max }\right)<x_{\max }$ for $0<a \leqslant \frac{1}{\sqrt{3}}$ (see Fig. 1).

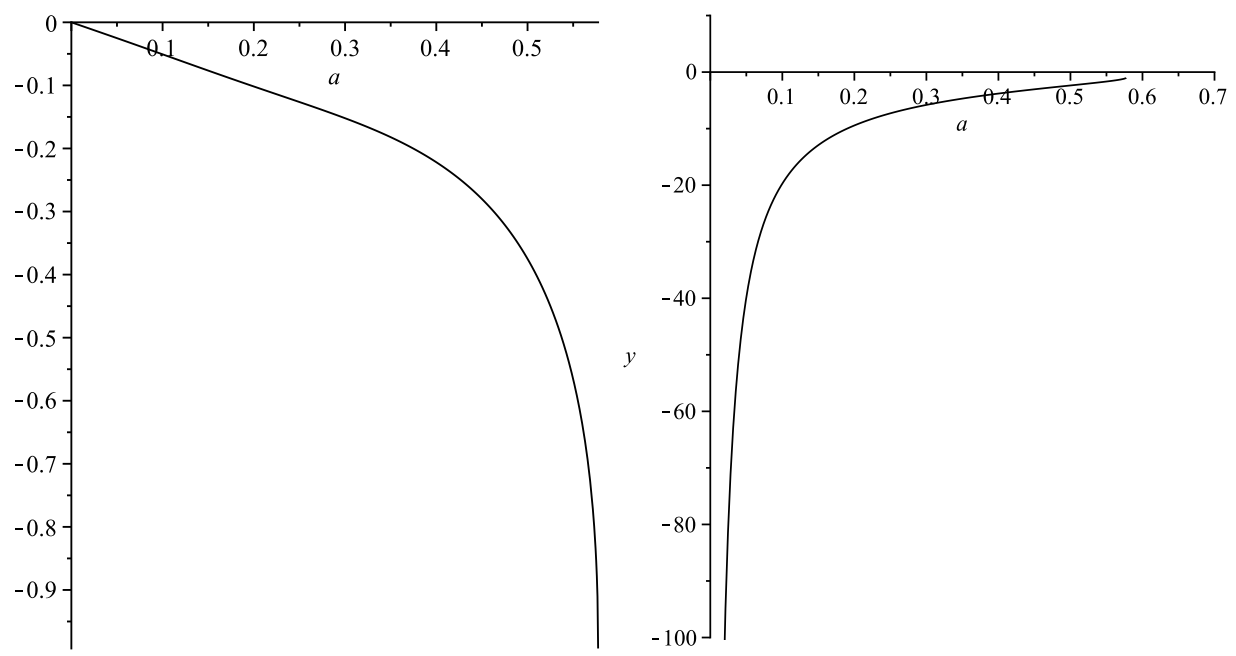

Fig. 1. Graphs of functions $g(a)=f\left(x_{\min }\right)-x_{\min }$ for $0<a \leqslant \frac{1}{\sqrt{3}}$ (on the left) and $g(a)=$ $f\left(x_{\max }\right)-x_{\max }$ for $0<a \leqslant \frac{1}{\sqrt{3}}$ (on the right)

From all this it follows

Theorem 4. In the case $G=$ gun for $k=2$ and $\lambda>0$ Hard-Core translation-invariant Gibbs measure is a unique.

The author is grateful to Professor U. A. Rozikov for useful discussions. 


\section{References}

[1] D.Galvin, J.Kahn, On phase transition in the hard-core model on $Z^{d}$, Combinatorics, Probability and Computing, 13(2004), 137-164.

[2] R.J.Baxter, Exactly Solved Models in Statistical Mechanics, Academic, London, 1982.

[3] F.Kelly, Loss networks, Ann. Appl. Probab., 1(1991), no. 3, 319-378.

[4] A.E.Mazel, Yu.M.Suhov, Random surfaces with two-sided constraints: an application of the theory of dominant ground states, J. Statist. Phys., 64(1991), 111-134.

[5] H.-O.Georgii, Gibbs Measures and Phase Transitions, De Gruyter Stud. Math., Vol. 9, Walter de Gruyter, Berlin, 1988.

[6] C.J.Preston, Gibbs States on Countable Sets, Cambridge Tracts Math., 68, Cambridge Univ. Press, Cambridge, 1974.

[7] Ya.G.Sinai, Theory of Phase Transitions: Rigorous Results, International Series in Natural Philosophy, vol. 108, Pergamon Press, Oxford-Elmsford, N.Y., 1982.

[8] Yu.M.Suhov, U.A.Rozikov, A hard-core model on a Cayley tree: an example of a loss network, Queueing Syst., 46(2004), no. 1/2, 197-212.

[9] R.M.Khakimov, Uniqueness of Weakly Periodic Gibbs Measure for HC-models. Math. Notes, 94(2013), no. 5, 139-143.

[10] J.B.Martin, U.A.Rozikov, Y.M.Suhov, A three state hard-core model on a Cayley tree, J. Nonlin. Math. Phys., 12(2005), no. 3, 432-448.

[11] U.A.Rozikov, Sh.A.Shoyusupov, Fertile three state HC models on Cayley tree, Theor. Math. Phys., 156(2008), no. 3, 1319-1330.

[12] G.R.Brightwell, P.Winkler, Graph homomorphisms and phase transitions, J. Combin. Theory Ser. B, $\mathbf{7 7}(1999)$, no. 2, 221-262.

[13] R.M.Khakimov, Translation-invariant Gibbs measures for fertile HC-models with three state on a Cayley tree, Theor. Math. Phys., DOI:10.4213/tmf8700.

[14] U.A.Rozikov, Gibbs measures on Cayley trees, World Scientific, 2013,

\section{Единственность трансляционно-инвариантной меры Гиббса для НС-моделей с четырьмя состояниями на дереве Кэли}

Рустам М. Хакимов

Рассмотрены плодородные Нагd-Соге (НС-модели) с параметром активности $\lambda>0$ и четырвмя состояниями на дереве Кэли порядка два. Известно, что существуют три типа таких моделей. В данной работе для каждой из этих моделей доказана единственность трансляиионноинвариантной меры Гиббс.

Ключевые слова: дерево Кэли, конфигурация, НС-модель, мера Гиббса, трансляционно-инвариантные меры. 